\title{
An Old Problem Resurfaces Nonlocally: Gamow's Liquid Drops Inspire Today's Research and Applications
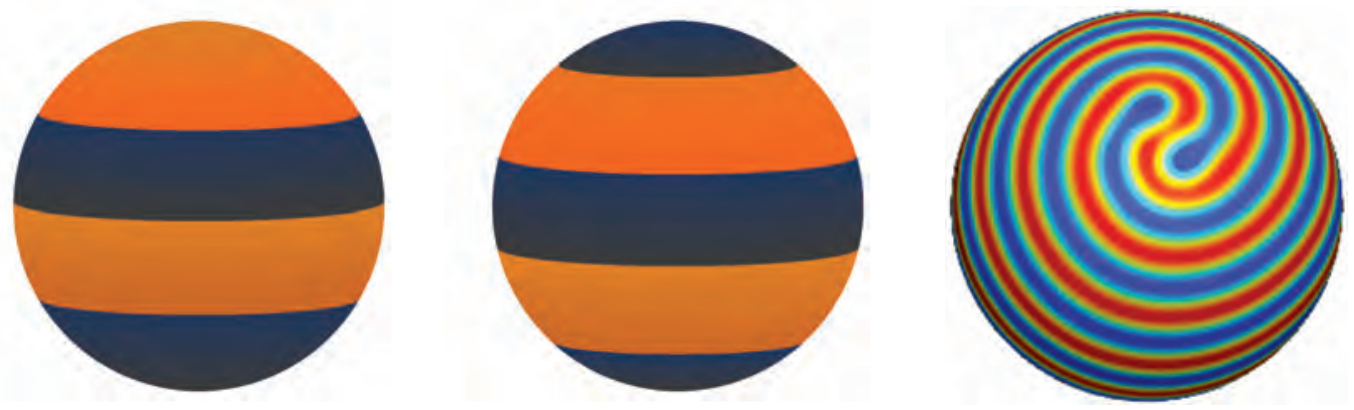

\section{Rustum Choksi, Cyrill B. Muratov, and Ihsan Topaloglu}

ABSTRACT. Gamow's simple liquid drop model of an atomic nucleus recently captured the attention of mathematicians and has inspired numerous advances and open questions in geometric and variational analysis.

"A mathematician friend of mine, the late S. Banach, once told me, 'The good mathematicians see analogies between theorems or theories; the very best see analogies between analogies.' This ability to see analogies between models for physical theories Gamow possessed to an almost uncanny degree."

-Stanislaw Ulam

Rustum Choksi is professor of mathematics at McGill University. His email address is rustum. choksi@mcgi11. ca.

Cyrill B. Muratov is professor of mathematics at New Jersey Institute of Technology. His email address is muratov@nj it. edu.

Ihsan Topaloglu is assistant professor of mathematics at Virginia Commonwealth University. His email address is iatopalog $1 \mathrm{u}$ @vcu.edu.

For permission to reprint this article, please contact: reprint-permission@ams.org.

DOI: http://dx.doi.org/10.1090/noti1598

\section{Gamow's Liquid Drop Model}

The 1920s may arguably be called the Golden Age of quantum mechanics. An explosive development of this emerging field of physics drew scores of aspiring researchers, one of whom was the young Russian theoretical physicist George Gamow (see Figure 1, page 1276 and sidebar on page 1278.) His name shot to fame when in 1928 , at the age of twenty-four, he published a paper in which he explained the phenomenon of alpha-decay as a quantum tunneling effect. In the words of Hans Bethe, the results of this paper constituted "the first successful application of quantum theory to nuclear phenomena." In the paper, Gamow also acknowledged a little help from Russian mathematician N. Kochin, admitting himself that he was "not good in mathematics."

In 1928 Gamow [1] made another important discovery that has become forever linked with his name. During his stay in Copenhagen with Niels Bohr in the fall of that year, Gamow conceived of what is now known as the liquid drop model of the atomic nucleus. This simple model, which was soon refined and further developed by Heisenberg, von Weizsäcker, and Bohr after the discovery of neutrons in 1932, treats the collection of protons and neutrons inside an atomic nucleus as an incompressible, uniformly charged fluid. With only a few fitting parameters and an assumption that the nuclei are spherical, this model was able to accurately predict the mass defect curve-the loss 


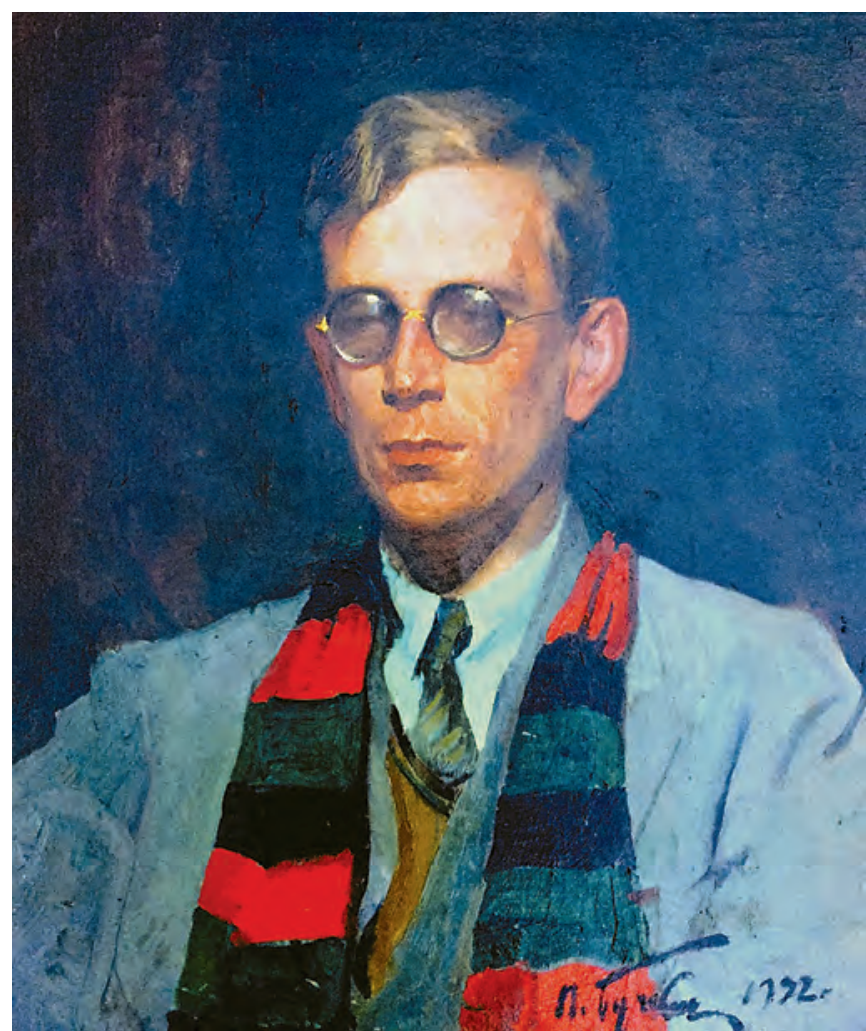

Figure 1. George Gamow (portrait, 1932). At the age of twenty-four, George Gamow introduced the liquid drop model that successfully explained the basic characteristics of the atomic nuclei and predicted nuclear fission as a result of an instability of large spherical nuclei with respect to nonspherical distortions.

of mass or energy when nucleons come together to bind in the nucleus as a function of the number of nucleons. Thus, indirectly, the model also predicted the spherical shape of most nuclei. The model's ultimate triumph came from explaining the phenomenon of nuclear fission in terms of an instability of large spherical nuclei with respect to nonspherical distortions (Bohr and Wheeler, 1939). The model has also been extensively used in astrophysics to describe exotic phases of nuclear matter at ultrahigh densities found in neutron stars (Baym, Bethe, and Pethick, 1971).

In the modern rendition of Gamow's liquid drop model the attractive short-range nuclear force gives rise to excess surface energy due to lower nucleon density near the nucleus boundary, while the presence of positively charged protons gives rise to a repulsive Coulombic force. Since the Coulomb energy of a proton in a nucleus is much smaller than its average kinetic energy determined by strong nuclear forces, to a good approximation the spatial distribution of charge in a nucleus is uniform. Therefore, mathematically the energy of a nucleus within the model may be written (up to shape-independent bulk terms and after a suitable nondimensionalization) as

$$
E(\Omega):=\operatorname{Per}(\Omega)+\frac{1}{8 \pi} \int_{\Omega} \int_{\Omega} \frac{1}{|x-y|} d x d y,
$$

where the nucleus $\Omega \subset \mathbb{R}^{3}$ is a measurable set with fixed volume $|\Omega|=m$. We refer to $m$ as "mass," which is a parameter proportional to the number of nucleons in a nucleus. $\operatorname{Per}(\Omega)$ is the perimeter of the set $\Omega$, i.e., a suitably generalized notion of the surface measure of $\partial \Omega .{ }^{1}$ The ground state of a nucleus with a given number of nucleons is then the minimizer of $E$, i.e., the set $\Omega$ that achieves the least energy,

$$
e(m):=\inf \{E(\Omega):|\Omega|=m\},
$$

for a given mass $m$.

$$
\begin{aligned}
& \text { a marriage (or } \\
& \text { rather divorce) } \\
& \text { of two older } \\
& \text { geometric } \\
& \text { problems }
\end{aligned}
$$

Ultimately, the purpose of this liquid drop model is to predict 1) the shape of nuclei, 2) the nonexistence of arbitrarily large nuclei, and 3 ) the existence of a nucleus with the least energy per nucleon (the element having the greatest nuclear binding energy). It is precisely the competition between the forces which try to minimize

the surface area of the nucleus and those which try to spread the nuclear charges apart that makes answering these questions nontrivial.

Gamow's liquid drop problem is a marriage (or rather divorce) of two older geometric problems:

(1) the Classical Isoperimetric Problem of minimizing the perimeter of a body with fixed mass $m$; and

(2) the Problem of the Equilibrium Figure of a selfgravitating fluid body of mass $m$.

For the first problem, whose roots go back to antiquity, Schwarz demonstrated the minimizing property of balls in 1884 for piecewise-smooth sets in three dimensions The complete solution was given in 1958 by De Giorgi, who showed that the unique minimizer of the perimeter functional among all measurable sets with fixed mass is given by a ball. Starting with Newton (1687), the second problem attracted the attention of many celebrated mathematicians. Assuming zero angular momentum, the total potential energy of a self-gravitating fluid body, represented by a measurable set $\Omega \subset \mathbb{R}^{3}$, is given, up to a constant, by

$$
-\int_{\Omega} \int_{\Omega} \frac{1}{|x-y|} d x d y, \quad|\Omega|=m
$$

where $-|x-y|^{-1}$ is the potential resulting from the gravitational attraction between two point masses at positions $x$ and $y$ in the fluid. Lyapunov (1886) made the first mathematical breakthrough by establishing that every regular minimizer of (3) is a ball. Poincaré commented on Lyapunov's proof in 1887 and went on to make the problem famous in his 1902 treatise Figures d'Equilibre d'une Masse Fluide. Almost twenty years later, Carleman (1919) showed that balls are indeed minimizers. Yet it was not until the work of Lieb (1977) that a complete solution based on strict Riesz rearrangement inequality

${ }^{1}$ See "WHAT IS...Perimeter?" in the October 2017 Notices. 
became available. Steiner symmetrization plays a central role in the analysis of both problems.

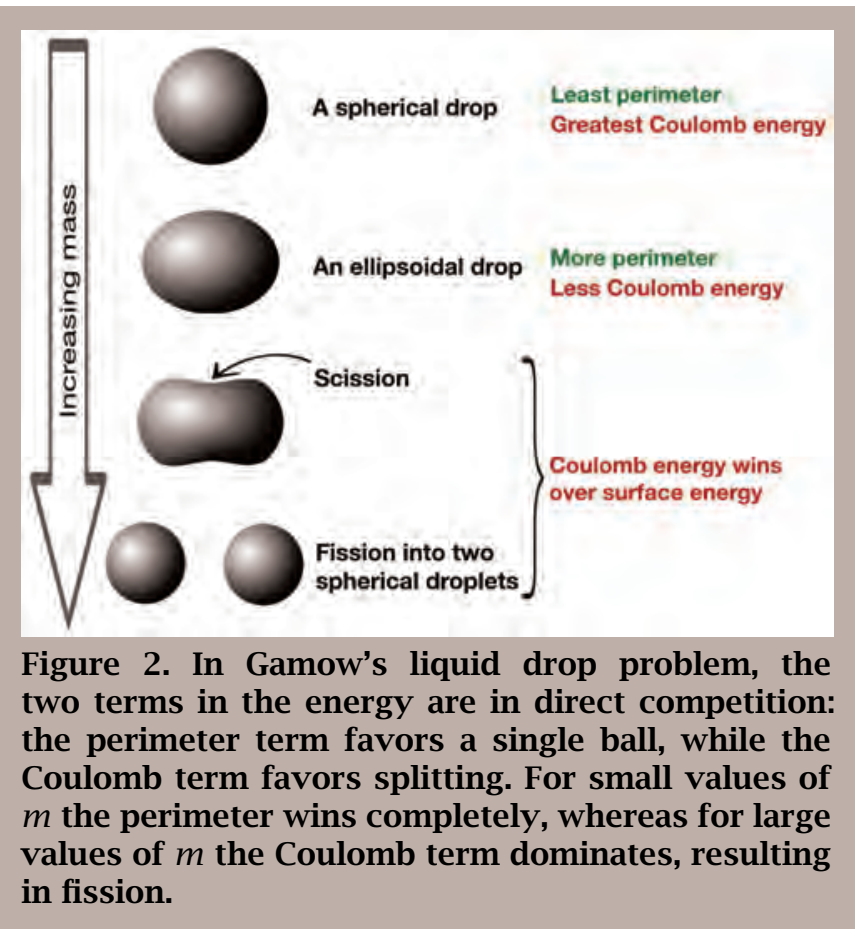

Thus, up to translations, the ball of mass $m$ is the unique minimizer in the two problems above. Gamow's liquid drop model, on the other hand, puts them in direct opposition, with balls being best for the perimeter term but worst for the Coulomb term. The relative strength of the two terms is controlled by the magnitude of $m$. One can readily see the effect of the size of $m$ as to which term dominates from a simple scaling argument. Namely, by looking at $\lambda \Omega$ for any $\lambda>0$ we see that

$$
\lambda^{-3} E(\lambda \Omega)=\lambda^{-1} \operatorname{Per}(\Omega)+\frac{\lambda^{2}}{8 \pi} \int_{\Omega} \int_{\Omega} \frac{1}{|x-y|} d x d y .
$$

Thus the energy per nucleon $\lambda^{-3} E(\lambda \Omega)$ is dominated by the perimeter term for small values of $\lambda>0$ and by the Coulomb term for large values of $\lambda>0$ (see Figure 2). Therefore, we would be led to expect that the unique solution (up to translations) of problem (2) is given by a ball when $m$ is small, whereas this problem does not have a solution when $m$ is large. Also, for fixed $\Omega$ there is a unique value of $\lambda$ at which the energy per nucleon (the negative of the binding energy) is minimum.

If one is to believe that Gamow's liquid drop model correctly captures the ground state configurations of the atomic nuclei, then nature tells us that the solution of the liquid drop problem is always given by a ball. In this case an explicit calculation gives the value of $m=$ $\frac{40 \pi}{3}\left(2^{1 / 3}+2^{-1 / 3}-1\right) \approx 44.1$, beyond which minimizers no longer exist. This critical value is such that one ball of mass $m$ has exactly the same energy as two balls with mass $\frac{1}{2} m$ infinitely far apart (Frank and Lieb, 2015). Under the same assumption, the minimum energy per unit mass is attained for $m=10 \pi \approx 31.4$.
Although Gamow's liquid drop model has been very well known among physicists, it is surprising that it did not receive much attention in the mathematics community until recently. In 2010 the liquid drop problem resurfaced in an asymptotic study by Choksi and Peletier [2], [3] of the Ohta-Kawasaki functional arising in a completely unrelated field of polymer physics. In fact, it is clear that the liquid drop problem represents a prime example of a problem of pattern formation from energetic competitions, which is an area driving much work in modern calculus of variations.

\section{Global Minimizers of the Liquid Drop Problem}

Global existence for minimizers of $E(\Omega)$ with $|\Omega|=m$ on unbounded domains is nontrivial because of the lack of compactness of minimizing sequences. What we know so far regarding the problem associated with $e(m)$ can be summarized by the following theorem. Here we consider the minimization problem (2) over all sets of finite perimeter (Caccioppoli sets).

Theorem 1. There exist constants $0<m_{0} \leq m_{1} \leq m_{2}$ such that:

(1) If $m \leq m_{1}$, then there exists a minimizer.

(2) If $m \leq m_{0}$, then the unique minimizer is a ball.

(3) If $m>m_{2}$, then there is no minimizer.

The result in this theorem, in this form, was first provided by Knüpfer and Muratov [6] in 2014. The existence of solutions for certain values of $m$ was already noted by Choksi and Peletier [2] in 2010 where the strict concavity of $e(m)$ for small values of $m$ was also established. Knüpfer and Muratov took a more direct approach and proved that for sufficiently small mass every minimizing sequence can be replaced by another minimizing sequence consisting of sets with uniformly bounded diameter. This uniform bound provided the necessary compactness for the minimizing sequence, and with the lower semicontinuity of $E(\Omega)$ with respect to the $L^{1}$-topology the existence of minimizers follows from the direct method of the calculus of variations. They also showed that minimizers of $E(\Omega)$ are so-called "quasiminimizers" of the perimeter functional and hence have smooth boundaries. Implementing the sharp quantitative isoperimetric inequality, proved by Fusco, Maggi, and Pratelli (2008), combined with the regularity, Knüpfer and Muratov proved that balls are, up to translations, unique minimizers of $E(\Omega)$ for sufficiently small mass. In order to prove that the problem does not have a solution for large masses, the authors first showed that minimizers are connected. However, they also showed that any connected set with sufficiently large mass can be divided into two large pieces by a suitable plane. Moving these pieces far apart from each other, they compared the energy of the transformed disconnected set with the original configuration and concluded the proof via a contradiction argument. 


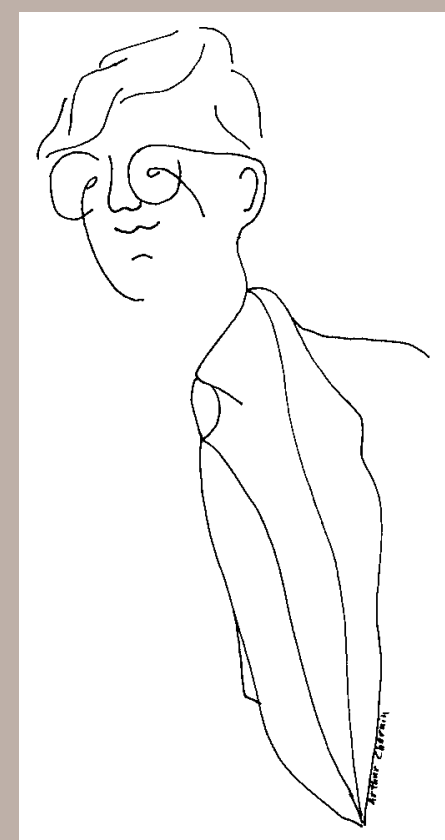

George Gamow

George Gamow (1904-1968) was a Russian-American theoretical physicist who introduced some of the most influential ideas to twentieth-century science. Born in 1904 in Odessa, Ukraine, Gamow graduated from Leningrad University in 1926, where together with fellow students L. Landau and D. Ivanenko he founded the group of the Three Musketeers to discuss the latest topics in theoretical physics, in addition to fooling around with friends and generally having a good time.

In the summer of 1928, Gamow undertook his first trip abroad on a four-month fellowship to the University of Göttingen, where he met many of the pioneers of quantum theory. Importantly, the trip also gave Gamow an opportunity to visit N. Bohr in Copenhagen and E. Rutherford at Cambridge, which was instrumental for the development of his quantum mechanical ideas in connection with nuclear phenomena.

\begin{abstract}
I shall never forget the first time he appeared in Göttingen-how could anyone who has ever met Gamow forget his first meeting with hima Slav giant, fair-haired and speaking a very picturesque German; in fact he was picturesque in everything, even in his physics.
\end{abstract}

\title{
-L. Rosenfeld
}

Gamow returned to the Soviet Union as a sort of celebrity. Yet the political atmosphere in the country had been rapidly changing, and Gamow felt it personally when in 1931 he was denied an exit visa to go to an international nuclear physics congress in Rome. From that time on, Gamow became obsessed with the desire to escape the confines of the Soviet Union, which in his mind had begun to interfere with the free movement of people and ideas across state borders. After an unsuccessful attempt to cross the Black Sea from Crimea into Turkey with his wife on a kayak, Gamow finally managed to obtain permission to leave the Soviet Union in 1933 for a conference, a trip from which he never returned. ${ }^{2}$ In 1934, Gamow settled at George Washington University in the United States, where he spent the next twenty-two years, the most productive period of his scientific career. He then moved to the University of Colorado, Boulder, where he worked until his death in 1968.

Gamow never received a Nobel Prize in physics, although his greatest achievements included the Big Bang theory, the theory of stellar structure and evolution, and a key insight into the nature of the genetic code. To the general public, however, Gamow is best known as the author of a popular science series, The Adventures of Mr. Tompkins, explaining the fundamental concepts of modern physics to millions of readers. ${ }^{a}$

${ }^{a}$ This issue's BookShelf (page 1327) features Gamow's popular text, One Two Three...Infinity: Facts and Speculations of Science.

Julin (2014) independently proved that for $m$ sufficiently small the ball of mass $m$ is the unique minimizer for $e(m)$, up to translations. His approach is to use a stronger version of the quantitative isoperimetric inequality. This version measures the difference between perimeters of a set and a ball of the same mass in terms of the oscillations of the boundary quantified by the $L^{2}$-norm of the difference of generalized outward normal vectors.

Independently, Lu and Otto (2014) also proved that for sufficiently large $m$, the energy $E(\Omega)$ does not admit a minimizer. By the subadditivity of $e(m)$,

$$
e(m) \leq e\left(m^{\prime}\right)+e\left(m-m^{\prime}\right), \quad 0<m^{\prime}<m,
$$

${ }^{2}$ The other members of the Three Musketeers group did not fare so well: L. Landau was arrested in 1938 and nearly died after one year in detention; D. Ivanenko was sentenced in 1935 to a three-year term in labor camps, substituted with a two-year exile in Tomsk after serving one year in Gulag; M. Bronshtein, another key member of the Leningrad group, was executed by NKVD in 1938. they obtain an upper bound of the form

$$
e(m) \leq C m, \quad m>m_{0} .
$$

In order to refine the lower bound of this scaling estimate, $\mathrm{Lu}$ and Otto prove a density estimate which states that a minimizer of $E(\Omega)$ cannot be thinner than order 1 in $m$, and combining this with the connectedness of minimizers, they obtain that for large values of $m$,

$$
\int_{\Omega} \int_{\Omega} \frac{1}{|x-y|} d x d y \geq C m \log m .
$$

Together with the upper bound this estimate yields a contradiction when $m$ is sufficiently large. More recently, via a very simple averaging technique applied to a cutting argument, Frank, Killip, and Nam (2016) proved that if $m>32 \pi \approx 100.53$, then the problem does not admit a minimizer. 


\section{The Liquid Drop Model on the Torus and the Ohta-Kawasaki Functional}

In the astrophysics context, one often considers the liquid drop model on the three-dimensional flat torus $\mathbb{T}_{\ell}^{3}$ of sidelength $\ell$. Specifically, for $\Omega \subset \mathbb{T}_{\ell}^{3}$ with $|\Omega|=m$ one considers the energy

$$
E_{\ell}(\Omega):=\operatorname{Per}\left(\Omega, \mathbb{T}_{\ell}^{3}\right)+\frac{1}{2} \int_{\mathbb{T}_{\ell}^{3}}\left(\chi_{\Omega}-\rho\right)(-\Delta)^{-1}\left(\chi_{\Omega}-\rho\right) d x,
$$

where $\operatorname{Per}\left(\Omega, \mathbb{T}_{\ell}^{3}\right)$ denotes the perimeter of $\Omega$ relative to $\mathbb{T}_{\ell}^{3}, \chi_{\Omega}$ is the characteristic function of $\Omega$, and $\rho=m / \ell^{3}$ is the density of the neutralizing background charge supplied by electrons. While this problem is well known in the studies of the structure of nuclear matter in neutron stars, it only recently received attention in the mathematics literature as the sharp interface version of the Ohta-Kawasaki functional

$$
\wp_{\ell}(u):=\int_{\mathbb{T}_{\ell}^{3}}\left(\frac{\varepsilon}{2}|\nabla u|^{2}+\frac{1}{4 \varepsilon}\left(1-u^{2}\right)^{2}+\frac{1}{2}(u-\bar{u})(-\Delta)^{-1}(u-\bar{u})\right) d x, \quad \frac{1}{\ell^{3}} \int_{\mathbb{T}_{\ell}^{3}} u d x=\bar{u},
$$

which is a diffuse interface version of the energy in (4) with the asymmetry parameter $\bar{u} \in(-1,1)$ fixed and $\varepsilon \ll 1$. The energy in (5) was introduced in 1986 by Ohta and Kawasaki as a simple model for self-assembly of diblock copolymer melts, even if its nuclear physics analog goes back all the way to the classical 1935 paper of von Weizsäcker. It can also be considered as a tool for numerical studies of (4).

Unlike the liquid drop problem posed on all space, the energy $E_{\ell}$ always admits a minimizer, and a question closely related to the liquid drop problem is what happens to those minimizers when $\ell \rightarrow \infty$ and/or $m \rightarrow 0$. Here the finiteness of the domain forces minimizers to always balance the effects of the perimeter and the Coulomb terms. In fact, numerically the putative global minimizers of the Ohta-Kawasaki functional appear to be periodic, with the interfaces within each intrinsic periodic cell resembling a constant mean curvature surface; e.g., a lamella, sphere, cylinder, double-gyroid, etc., as in Figure 3. Thus numerics suggest a separation of the effects of the perimeter and the nonlocal term: the latter sets an intrinsic periodicity, while the former dictates the interface structure within the period cell. This separation has been exploited in a number of papers by Ren and Wei $(2007,2008,2009)$ and by Cristoferi (2016) for constructing critical points of variants of (4) perturbatively. To gain further insights into this separation, an asymptotic analysis was presented by Choksi and Peletier [2], [3] and by Knüpfer, Muratov, and Novaga [4] for the droplet regimes wherein $\rho \rightarrow 0$. Here, Gamow's liquid drop model (on all $\mathbb{R}^{3}$ ) emerged as the leading-order energy describing the shape of a single droplet, ignoring the next-order effects of the neighboring droplets.

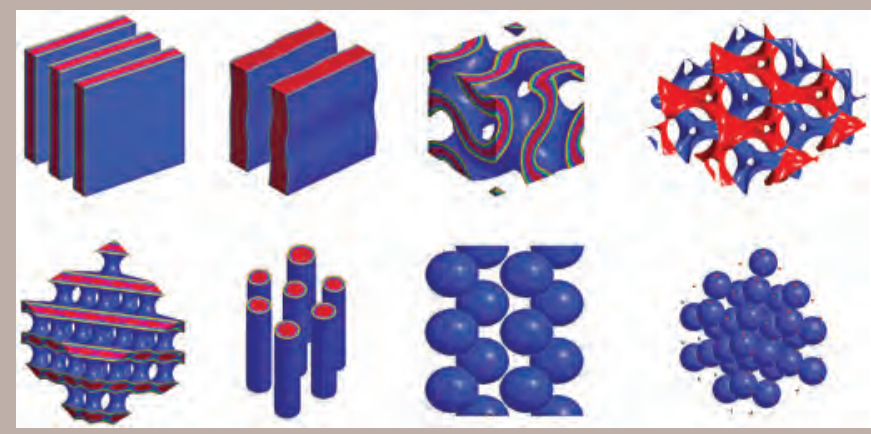

Figure 3. Level sets of the computed local minimizers of Ohta-Kawasaki energy $\mathscr{\wp}_{\ell}$ in (5) as the value of $\bar{u}$ is varied.

While one may conjecture that it is possible to choose $m_{0}=m_{1}=m_{2}$, based on physical evidence, to date it remains open to prove or disprove whether any (or all) of the constants $m_{i}, i=0,1,2$, may be pairwise equal. One result in that direction was obtained by Knüpfer, Muratov, and Novaga [4] in 2016, who show that the set

$$
\mathcal{I}=\{m>0: e(m) \text { is attained }\}
$$

is a closed (hence compact) subset of $(0, \infty)$.
They establish this by proving Lipschitz continuity of $e(m)$ on compact subsets of $(0, \infty)$ combined with the $B V$-compactness of sequences of generalized minimizers. Here a generalized minimizer of $E(\Omega)$ refers to a finite collection of sets of masses $m_{j}$ adding up to $m$, where each set is a minimizer for $e\left(m_{j}\right)$. Around the same time, Frank and Lieb (2015) obtained the same result, using a diameter bound of the form diam $\Omega \leq C m$ for minimizers of $E(\Omega)$, along with a concentration-compactness lemma, 

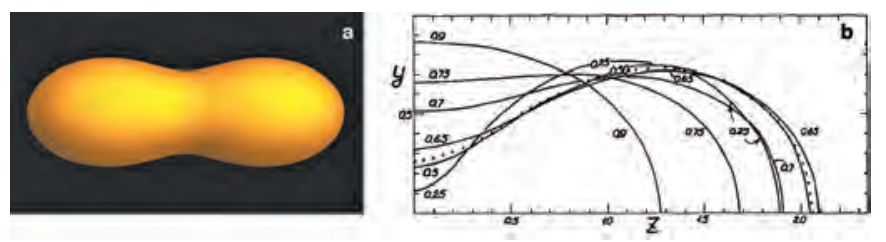

Figure 4. Dumbbell-shaped critical points of the liquid drop model for several values of the fissility parameter $\alpha=m /(40 \pi)$ obtained from the numerical solution of (6) by Strutinsky, Lyashchenko, and Popov (1963): a three-dimensional schematic (a), profile cross-sections in the first quadrant (b).

particularly well-suited to the liquid drop problem, for a sequence of sets of uniformly bounded perimeter. Together with strict concavity of $e(m)$ for $m \leq 4 \pi$, these results also yield the existence of a minimizer in this range of masses.

Finally, referring back to the third purpose of the liquid drop model, existence of the tightest bound nucleus was established in 2016 by Knüpfer, Muratov, and Novaga [4], who show that there exists $m^{*} \in \mathcal{I}$ such that

$$
f^{*}:=\inf _{m \in \mathcal{I}} f(m)=f\left(m^{*}\right), \quad f(m):=\frac{e(m)}{m} .
$$

The proof of this fact follows from Lipschitz continuity of the energy-per-particle $f(m)$ on the set $\left[m_{0}, \infty\right)$ combined with compactness of the set $\mathcal{I}$. In fact, the analysis further shows that the quantity $f^{*}$ appears as the leading order term in the asymptotic behavior of uniform energy density of minimizers of $E_{\ell}(\Omega)$ as mass $m \rightarrow 0$ and domain size $\ell \rightarrow \infty$. Frank and Lieb (2015) also prove the same existence result for $f(m)$ by relaxing the mass constraint $|\Omega|=m$ and considering $\inf _{0<|\Omega| \leq m}(E(\Omega) /|\Omega|)$.

\section{Critical Points and Local Minimizers of the Liquid Drop Problem}

One of the main physical motivations in the studies of Gamow's liquid drop model was to compute the minimal energy required for nuclear fission. Bohr and Wheeler (1939), following the suggestion of Meitner and Frisch (1939), were the first to analyze the critical points of the energy in (1). In particular, they wrote down the associated Euler-Lagrange equation

$$
2 \mathcal{H}(x)+\frac{1}{4 \pi} \int_{\Omega} \frac{1}{|x-y|} d y=\lambda,
$$

where $\mathcal{H}(x)$ denotes the mean curvature of $\partial \Omega$ (positive if $\Omega$ is convex) and $\lambda$ is a Lagrange multiplier. Clearly, a ball of mass $m$ is always a solution of (6). However, as was shown numerically in a series of papers from the 1940s through the 1970s, many types of nonspherical solutions to (6) are possible, including ovoid, dumbbell, pearshaped, and toroid nuclei. In fact, when the ball of mass $m$ passes the stability threshold of $m=40 \pi \approx 125.66$, it bifurcates into a family of spheroids, first identified by Bohr and Wheeler (1939), using formal bifurcation analysis near the distortion instability. Numerical continuation studies of those solutions into the subcritical mass region

were carried out by several authors, but, surprisingly, only one well-converged numerical study, by Strutinsky, Lyashchenko, and Popov (1963), appears to exist to date; see Figure 4.

Although mathematically it is not in the literature whether suitable nonspherical distortions of balls are critical points, using Lyapunov-Schmidt type reduction methods Ren and Wei $(2011,2014)$ obtained existence of solutions to the Euler-Lagrange equation which are almost of the shape of single and double tori. However, these more exotic patterns, as shown by Ren and Wei (2017), are highly unstable in the sense of the second variation of the energy. Earlier, oval-shaped solutions in a two-dimensional version of the problem and spherical shell solutions in three dimensions were constructed by Ren and Wei (2009), with some numerical studies of spherical shell and toroidal solutions previously carried out by Wong (1973).

Obtaining quantitative information about (local) minimizers through stability is the approach taken by Bonacini and Cristoferi (2014), based on the explicit calculations of the second variation of $E(\Omega)$ obtained by Muratov (2002) and Choksi and Sternberg (2007). They generalize the result of Acerbi, Fusco, and Morini (2013) for bounded domains to the unbounded setting of the liquid drop problem and prove that strictly stable critical points, i.e., sets for which the first variation of $E(\Omega)$ vanishes and the second variation of $E(\Omega)$ is strictly positive-definite, are local minimizers. That is, these patterns minimize $E(\Omega)$ with respect to competitors close in the $L^{1}$-topology. Applying this result to the ball of mass $m$, they conclude that the ball is an isolated local minimizer if $m<40 \pi$. Finally, very recently Julin (2016) proved that every local minimizer with sufficiently small mass and perimeter is a ball, using the characterization of compact almost constant mean curvature surfaces by Ciraolo and Maggi (2015).

At the heart of these criticality and stability results lies the regularity of the boundaries of local minimizers. As was first proved by Sternberg and Topaloglu (2011), local minimizers of $E_{\ell}(\Omega)$ have $C^{3, \alpha}$ boundaries by using classical geometric measure theoretic arguments from regThe simplicity ularity of minimal surfaces. More recently, Julin and Pisante

of Gamow's liquid drop model conceals the true richness. (2015) demonstrated that the boundaries of local minimizers are in fact of class $C^{\infty}$, and Julin (2016) established analyticity of the boundary of regular critical points. Since the regularity arguments are local arguments, they immediately apply to local (and global) minimizers of the liquid drop problem on the whole of $\mathbb{R}^{n}$. Furthermore, any critical point of the energy in the $L^{1}$-topology is what is known as a $\Lambda$-minimizer of the perimeter and hence is also regular. 

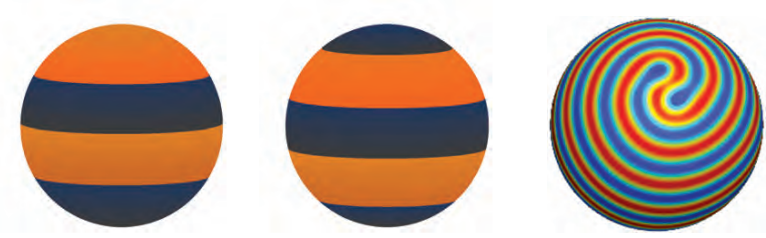

Figure 5. Examples of critical points of the liquid drop model on the sphere. In the first two panels, axisymmetric configurations with three and four interfaces are shown. The third panel is the result of a hybrid numerical simulation by Shahriari, Ruuth, and Choksi, where a spiraling pattern achieves a stable low energy state.

\section{Extensions and Related Problems}

The simplicity of Gamow's liquid drop model conceals the true richness of behaviors exhibited by the solutions of this geometric variational problem. At the same time, it is clear that the main ingredients of Gamow's model, namely, the competition between the short-range attractive and long-range repulsive forces, are not unique to that model and should be expected to produce interesting behaviors in a variety of contexts, both mathematically and physically.

The simplest natural mathematical generalization of Gamow's liquid drop model is to extend it to other space dimensions. Considering $\Omega \subset \mathbb{R}^{n}$ with $n>3$ amounts to replacing the Newtonian kernel in (1) with a Riesz kernel $|x-y|^{-\alpha}$, where $\alpha=n-2$. For this problem, it was shown by Knüpfer and Muratov [5], [6] that minimizers still exist for all sufficiently small masses and that minimizers for yet smaller masses are balls when $n \leq 7$. Julin (2014) extended this result to all dimensions by removing the reliance on the classical regularity of minimal surfaces. Yet, the arguments leading to nonexistence no longer apply, and it is an open problem whether or not minimizers to the liquid drop problems, which must then be nonspherical as it is always better to split one ball into two identical balls far apart for $m \gg 1$, exist for arbitrarily large masses when $n \geq 4$. The absence of such a nonexistence result also prevents proving existence of minimizers with optimal energy per unit mass.

On the other hand, Gamow's liquid drop problem becomes trivial for $n<3$, since in that case the fundamental solution of Laplace's equation is no longer positive, and minimizers of the liquid drop problem do not exist for any value of $m>0$. The problem is still meaningful if posed instead on a compact Riemannian manifold, and results for global minimizers on a two-dimensional flat torus or a two-dimensional sphere are available; see Sternberg and Topaloglu (2011) and Topaloglu (2013). Locally minimizing axisymmetric critical patterns on the sphere as those in Figure 5 have also been investigated by Choksi, Topaloglu, and Tsogtgerel (2015). The onedimensional periodic case is considerably simpler and was solved completely by Ren and Wei (2003). Another alternative is to consider the problem on the whole of
$\mathbb{R}^{n}$ but replace the Newtonian potential with a general Riesz kernel with $0<\alpha<n$. In fact, the case $n=2$ and $\alpha=1$ arises in the physical modeling of charge condensation in high- $T_{c}$ superconductors. In this case existence of minimizers with small masses was again obtained by Knüpfer and Muratov [5], [6], where minimality of balls of small masses was shown for $n=2$ and for all $3 \leq n \leq 7$ and $\alpha<n-1$. The latter result was extended to $n \geq 8$ by Bonacini and Cristoferi (2014). The case of $n \geq 3$ and $n-1 \leq \alpha<n$ was settled by Figalli, Fusco, Maggi, Millot, and Morini (2015), who also considered a nonlocal generalization of the perimeter functional to fractional perimeter. We note that the latter has been the subject of considerable attention recently, starting with the work of Caffarelli, Roquejoffre, and Savin (2010), and gives rise to nonlocal minimal surfaces. In contrast with Gamow's model, however, the fractional perimeter represents a nonlocal generalization of the attractive potential that keeps the "liquid drop" together, and, therefore, it alone does not produce the phenomenology of Gamow's liquid drop problem. Back to the liquid drop problem with a Riesz kernel, a nonexistence result for large masses is available for $\alpha<2$ (Knüpfer and Muratov [5], [6]). Again, it is an open problem whether or not large nonspherical minimizers could exist for $n \geq 3$ and $\alpha \geq 2$. Lastly, in the special case $n=2$ and $\alpha$ sufficiently small, a complete solution of the liquid drop problem with a Riesz potential was given by Knüpfer and Muratov [5] in 2013. The obtained solution agrees with the physical conjecture about the structure of the minimizers of the classical Gamow's liquid drop problem mentioned earlier.

Moving on to further extensions, we note that Coulombic repulsive forces naturally arise in a number of other physical contexts. Perhaps the best known example goes back to Lord Rayleigh (1882), who considered equilibrium shapes of charged liquid drops that are perfect conductors. The associated energy functional may be written in a nondimensional form as

$$
E(\Omega):=\operatorname{Per}(\Omega)+\frac{\lambda}{8 \pi} \inf _{\mu \in \mathcal{P}(\Omega)} \int_{\Omega} \int_{\Omega} \frac{d \mu(x) d \mu(y)}{|x-y|},
$$

where $\Omega \subset \mathbb{R}^{3}$ with $|\Omega|=m$, as before, and the infimum is taken over all probability measures supported on $\Omega$. One would naturally be led to believe that the minimization problem for (7) would behave quite similarly to the Gamow liquid drop problem. Yet, Goldman, Novaga, and Ruffini (2015) showed that, surprisingly, the minimization problem for (7) does not admit minimizers for any $m>0$ and $\lambda>0$. Even worse, the problem does not exhibit local minimizers in any reasonable sense, indicating that the energy in (7) does not provide a complete physical picture as a model of charged liquid drops, as shown by Muratov and Novaga (2016). The reason for this behavior is a kind of incompatibility between the sets seen by the perimeter, which have finite two-dimensional Hausdorff measure of the measure theoretic boundary, and sets of positive capacity, which may have positive Hausdorff measure of dimension $1<d<2$. This phenomenon also gives rise to singular solutions of the associated EulerLagrange equation, such as the famous Taylor cone with 


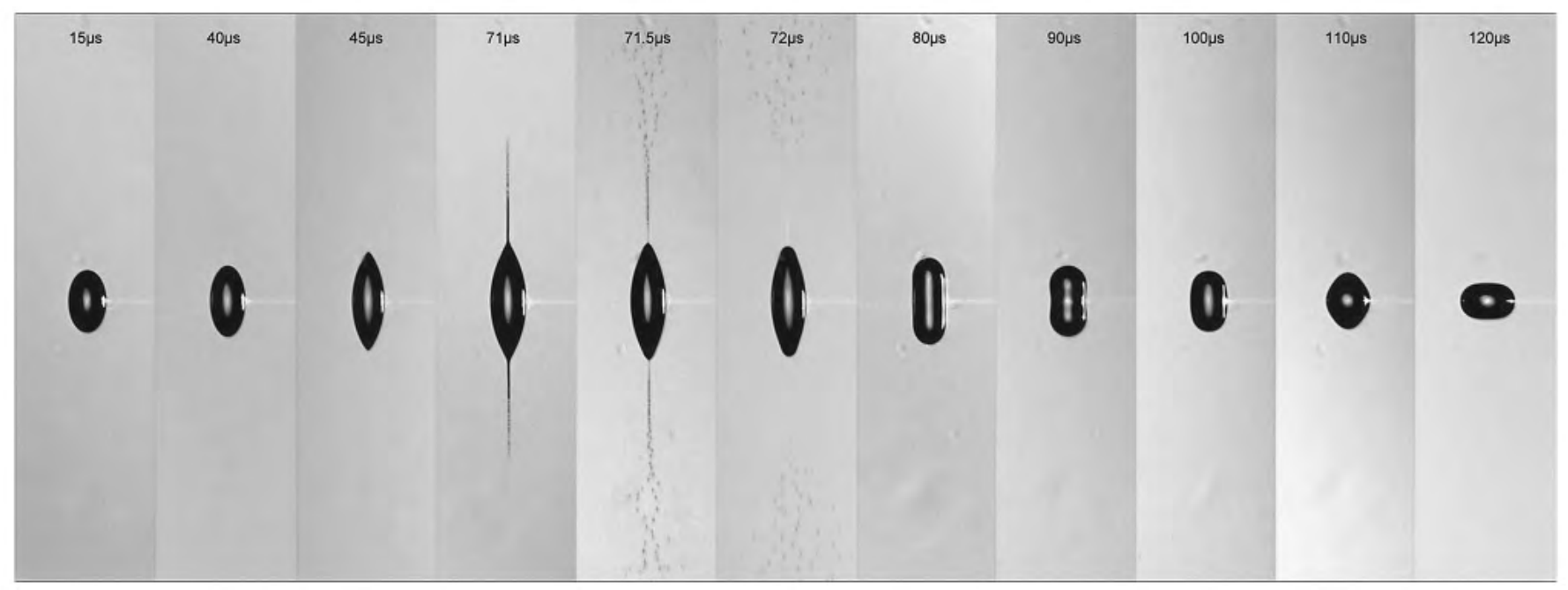

Figure 6. A sequence of snapshots of a levitating charged liquid drop reaching the threshold of the charge-driven instability as the liquid in the drop evaporates.

an opening half-angle of $\approx 49.3^{\circ}$, obtained by Taylor (1962). Further regularity assumptions on the set, such as convexity of $\Omega$, yield existence, as was shown by Goldman, Novaga, and Ruffini (2016). It is also clear that the model in (7) is quite sensitive to the space dimensionality. In fact, a complete solution of a variant of the minimization problem for (7) in which the set $\Omega$ is flat, i.e., $\Omega=D \times\{0\}$, where $D \subset \mathbb{R}^{2}$, with $|D|=m$, is a closed set and the perimeter is replaced by the one-dimensional Hausdorff measure of $\partial D$, has been obtained by Muratov, Novaga, and Ruffini (2017), who prove that the minimizer with "mass" $m$ and "charge" $\sqrt{\lambda}$ exists if and only if $\lambda \leq 4 m / \pi$ and is given by a disk. Note that here a single disk remains a minimizer against splitting into two equal disks for all $\lambda<4 m \sqrt{2} / \pi$. Thus, the scenario of existence failure is different from the one hypothesized for Gamow's liquid drop model: instead of splitting into two disks, a single disk breaks into one big disk with a certain amount of charge and many tiny disks that carry the excess charge to infinity to reduce energy for $\lambda>4 m / \pi$. We note that in physical experiments the failure of existence of minimizers in the three-dimensional charged liquid drop model manifests itself spectacularly via thin jets of electrified liquid emitted from the droplet as its smoothness is lost, as in Figure 6. Smoothness is then restored as a significant portion of charge escapes the drop via the jet.

\section{Conclusion}

Gamow's liquid drop model is indeed a very simple model for a highly complex system. However, its beauty is really in tune with the famous quote attributed to Albert Einstein: Everything should be made as simple as possible, but not simpler. While it was initially posed to describe nuclear structures, the fact that it encapsulates a rather ubiquitous competition of short- and long-range effects is behind a universality, with the liquid drop model's phenomenology shared by many other systems operating at very different length scales: from femtometer nuclear scale to nanoscale in condensed matter systems, to centimeter scale for fluids and autocatalytic reactiondiffusion systems, all the way to cosmological scales. It has generated much recent attention by mathematicians and continues to this day to entice and challenge us with its beguilingly simple yet rich structure.

\section{References}

[1] G. Gamow, Mass defect curve and nuclear constitution, Proc. R. Soc. Lond. A 126 (1930), no. 803, pp. 632-644.

[2] R. Choksi and M. A. Peletier, Small volume fraction limit of the diblock copolymer problem: I., Sharp-interface functional, SIAM J. Math. Anal. 42 (2010), no. 3, pp. 1334-1370. MR 2653253 (2011j:49092)

[3] , Small volume-fraction limit of the diblock copolymer problem: II., Diffuse-interface functional, SIAM J. Math. Anal. 43 (2011), no. 2, pp. 739-763. MR 2784874(2012e:49097)

[4] H. KNüPfER, C. B. MurAtov, and M. NovagA, Low density phases in a uniformly charged liquid, Comm. Math. Phys. 345 (2016), no. 1, pp. 141-183. MR 3509012

[5] H. KNÜPFER and C. B. MuRATOV, On an isoperimetric problem with a competing nonlocal term I: The planar case, Comm. Pure Appl. Math. 66 (2015), no. 7, pp. 1129-1162. MR 3055587

[6] , On an isoperimetric problem with a competing nonlocal term II: The general case, Comm. Pure Appl. Math., 67 (2014), no. 12, pp. 1974-1994. MR 3272365

\section{Image Credits}

Figure 1 (portrait of George Gamow, 1932) courtesy of Elfriede and Igor Gamow.

Figure 2 created by the authors.

Hand-drawn portrait of George Gamow courtesy of A. D. Chernin, "Gamow in America: 1934-1968 (On the ninetieth anniversary of G. A. Gamow's birth)," Physics-Uspekhi, vol. 37, pp. 791-802, 1994, reprinted with permission. 
Figure 3 simulations by J. F. Williams, discussed in Choksi, Peletier, and Williams (2009).

Figure $4 \mathrm{a}$ created by the authors.

Figure 4b reprinted from Nuclear Physics 46, V. M. Strutinsky, N. Ya. Lyashchenko, and N. A. Popov, "Symmetrical shapes of equilibrium for a liquid drop model," pp. 639-659, JulySeptember 1963, with permission from Elsevier.

Figure 5 courtesy of B. Shahriari, R. Choksi, I. Topaloglu, and G. Tsogtgerel, "Axisymmetric critical points of a nonlocal isoperimetric problem on the two-sphere," ESAIM: Control, Optimisation and Calculus of Variations, vol. 21, no. 1, pp. 247-270, 2015, reprinted with permission.

Figure 6 courtesy of E. Giglio, B. Gervais, J. Rangama, B. Manil, B. A. Huber, D. Duft, R. Müller, T. Leisner, and C. Guet, "Shape deformations of surface-charged microdroplets," Physical Review E 77, 036319, 2008. Copyright 2008 by the American Physical Society. dx.doi.org/10.1103/PhysRevE.77 .036319 . Reprinted with permission.

\section{ABOUT THE AUTHORS}

Rustum Choksi focuses his mathematical research on the calculus of variations and its applications to pattern formation. Since he was a child, he has been a passionate lover of classical music, from chamber music to opera. He also devotes too much time and money to collecting minerals, in particular agates with their alluring and infinite-dimensional pattern morphology.

Cyrill B. Muratov is interested in the emergence of spatial complexity from basic constitutive laws. He uses a combination of rigorous mathematical analysis, formal asymptotics, and numerical simulations to understand complex physical phenomena arising in science and engineering.

Since high school, Ihsan Topaloglu has been fascinated by the connection between mathematics and physics. He now studies pattern formation in models of materials and biological aggregations through geometric variational problems.

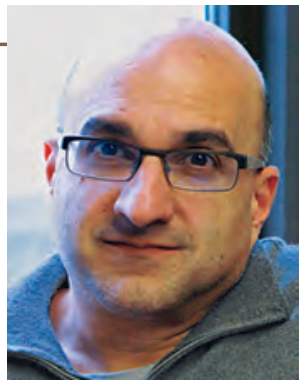

Rustum Choksi

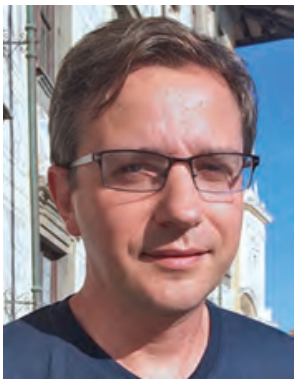

Cyrill B. Muratov

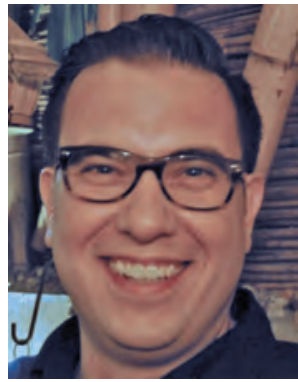

Ihsan Topaloglu

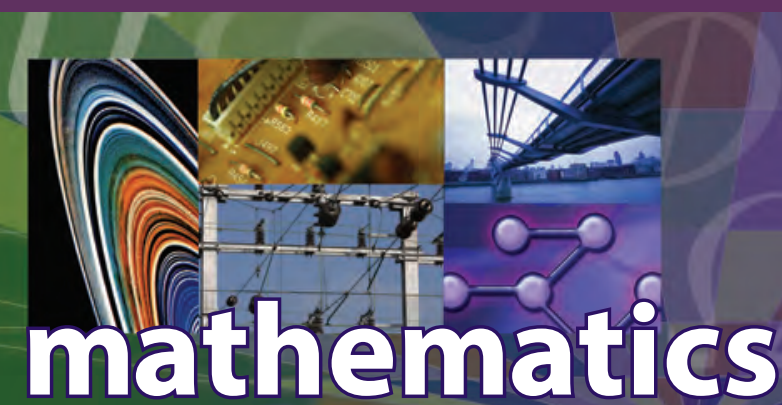

\section{LANGUAGE OF THE SCIENCES}

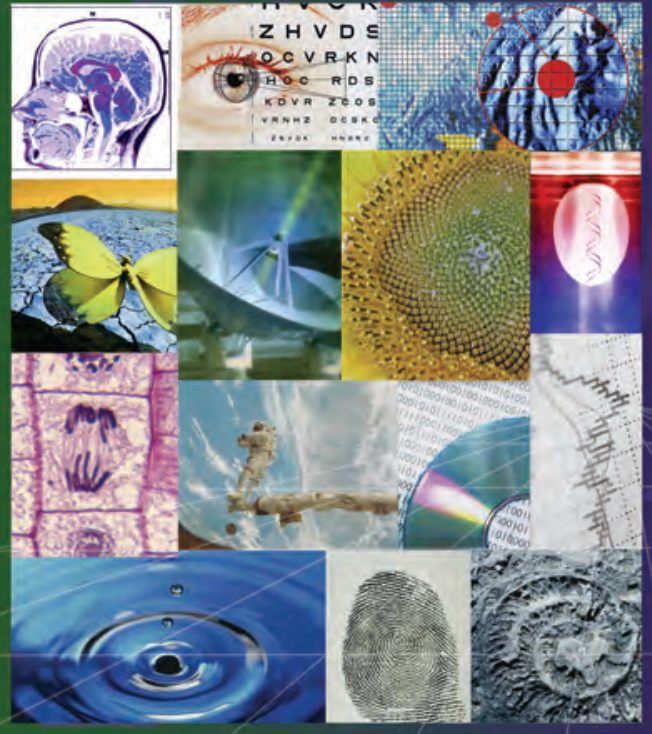

engineering

astronomy robotics

genetics

biology

climatology forensics

statistics finance

computer science

physics

neuroscience

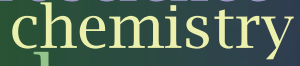

ge 010 gy

biochemistry

ecology

molecular biology 